\title{
Optical Burst Control Algorithm for Reducing the Effect of Congestion Reaction Delay
}

\author{
Myungsik Yoo and Junho Hwang \\ School of Electronic Engineering, Soongsil University, Seoul, Korea \\ $\{$ myoo, jhwang\} @ssu.ac.kr
}

\begin{abstract}
To address the burst loss problem in OBS network, we propose a new optical burst control algorithm, which estimates the future burst traffic condition to eliminate the effect of congestion reaction delay. Through the simulations, we verify that the proposed algorithm outperforms other existing burst control algorithms.
\end{abstract}

Keywords: Optical Burst Switching, Congestion Control, Congestion Reaction Delay, Estimation.

\section{Problem Statement}

In optical burst switched (OBS) [1] network, a data burst is assembled at ingress edge router, and then is forwarded to core router for delivery to its destination. When the core router receives data bursts more than it can handle, the congestion control algorithm detects the congestion, and then informs the corresponding ingress edge routers. Upon receiving congestion feedback information, the ingress edge router reacts against the congestion by reducing burst transmission rate. Thus, as can be seen in Fig. 1, it takes round trip time (RTT) for a core router to detect the congestion, and then to receive the reduced burst flow from the ingress edge router, which is called the congestion reaction delay. It means that a huge amount of data bursts might be lost during the congestion reaction delay if a long-lasting burst congestion presents.

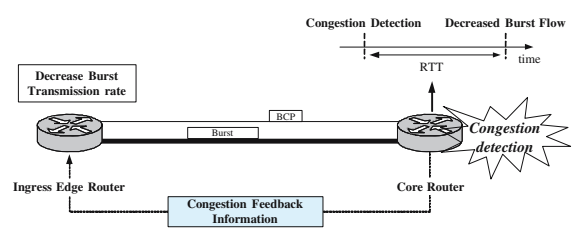

Fig. 1. Effect of congestion reaction delay

One can handle the burst loss problem using the burst congestion control algorithm based on the current traffic information [2]. Let $\tau, D(t)$ and $L_{t h}$ denote the sampling period, the amount of data burst received in $[t, t+\tau]$ and the 
congestion detection threshold, respectively. Then, this congestion control algorithm makes the decision, if $D(t) \geq L_{t h}$, then congestion condition. Otherwise, normal condition. Due to the congestion reaction delay, this approach suffers from a high burst loss when the long-lasting burst congestion presents.

The burst loss problem can be handled by the burst congestion estimation algorithm based on a single statistic [3. Let $D_{\text {avg }}(t)$ denote the weighted moving average of the samples at time $t$, where each sample $D(t)$ indicates the amount of data burst received in a sample period $[t, t+\tau]$. Then, $D_{\text {avg }}(t)$ is obtained by

$$
D_{\text {avg }}(t)=(1-\alpha) \times D_{\text {avg }}(t-1)+\alpha \times D(t), 0<\alpha<1 .
$$

The congestion estimation algorithm based on a single statistic makes the decision, if $D_{\text {avg }}(t) \geq L_{t h}$, then congestion condition. Otherwise, normal condition. If $\tau$ is shorter than RTT, it suffers from the congestion reaction delay penalty. $\tau$ can be set to $R T T$ to eliminate the congestion reaction delay, but it leads to average down the traffic characteristics shown in shorter intervals than $\tau$.

\section{Burst Control Algorithm with Long-Term Estimation: BCA-LTE}

The proposed congestion control algorithm utilizes multiple statistics on burst traffic, which are measured over the various sampling periods. Let $k$ denote segment constant where $k$ is an arbitrary positive integer, and divide RTT into $2^{k}$ small segments where each segment spans $R T T / 2^{k}\left(=R T T \times 2^{-k}\right)$. We define the smallest segment as $\tau_{2^{-k}}$, which is the smallest burst traffic sampling period for estimation. For every $\tau_{2^{-k}}$, obtain $D_{2^{-k}}(t)$ and get $D_{a v g, 2^{-k}}(t)$ by applying Eqn. (11).

Now, define another sampling period, where the samples are taken for every two $\tau_{2^{-k}}$. This sampling period spans $R T T / 2^{(k-1)}\left(=R T T \times 2^{-(k-1)}\right)$, which is denoted as $\tau_{2^{-(k-1)}}$. For every $\tau_{2^{-(k-1)}}$, obtain $D_{2^{-(k-1)}}(t)$ and get $D_{a v g, 2^{-(k-1)}}(t)$ by applying Eqn. (11).

By increasing the sampling period by a factor of two until it reaches to RTT, one can define $(k+1)$ different sampling periods $\left(\tau_{2^{-i}}, i=k, k-1, \cdots, 0\right)$ and obtain $(k+1)$ statistics $\left(D_{\text {avg }, 2^{-i}}(t), i=k, k-1, \cdots, 0\right)$ for each sampling period. $D_{\text {avg, } 2^{-i}}(t)$ reflects how burst traffic changes in short and long terms.

One can summarize all statistics into a single estimation point using Eqn. (2), where EST stands for estimated burst traffic at future time $t+R T T$. In Eqn. (2), $E S T_{k}(t+R T T)$ is a predicted burst traffic condition at $t+R T T$, where the statistics on longer sampling periods are reflected more.

$$
\begin{aligned}
E S T_{0}(t+R T T) & =D_{\text {avg } 2^{-k}}(t) \\
E S T_{1}(t+R T T) & =\alpha \times E S T_{0}(t+R T T)+(1-\alpha) \times D_{a v g, 2^{-(k-1)}}(t), \\
E S T_{2}(t+R T T) & =\alpha \times E S T_{1}(t+R T T)+(1-\alpha) \times D_{a v g, 2^{-(k-2)}}(t), \\
\vdots & \\
E S T_{k}(t+R T T) & =\alpha \times \operatorname{EST}_{(k-1)}(t+R T T)+(1-\alpha) \times D_{a v g, 2^{-(0)}}(t) .
\end{aligned}
$$


In order to have more estimation points in a RTT period, we introduce the tier and define $N$ and $n$ as the number of tiers and tier index, respectively, where $n=0,1, \cdots, N-1$. Tier[0] becomes a timing reference to other $N-1$ tiers. The timing difference between two neighboring tiers equals to $R T T / N$. Thus, each tier starts its RTT at $t+R T T \times n / N$. Each tier runs the estimation algorithm as in Eqn. (2) and generates its own estimation point. By having $N$ tiers, one can get $N$ estimation points over a RTT period. At every estimation point that each tier generates, the congestion control algorithm makes decision, if $E S T_{k}(t+R T T) \geq L_{t h}$, then congestion condition. Otherwise, normal condition.

\section{Simulation Results and Discussion}

For performance evaluation, we consider a simple network topology as shown in Fig. 2. We assume a simple burst assembly mechanism, where the burst rate is controlled by adjusting the burst size to be assembled. For the comparison purpose, we evaluate the performance of three algorithms: the proposed algorithm (indicated as BCA-LTE), the algorithm based on a single statistic (indicated as SINGLE) and the algorithm without any burst congestion control (indicated as $\mathrm{w} / \mathrm{o} \mathrm{CC})$.

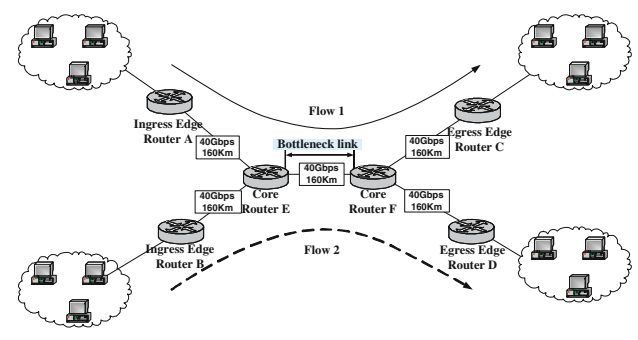

Fig. 2. Simulation topology

Table 1 shows that BCA-LTE algorithm outperforms SINGLE algorithm in terms of estimation accuracy. In fact, BCA-LTE algorithm achieves about $95 \%$ of accuracy. These results prove that the estimation based on multiple statistics helps to improve the accuracy of congestion detection.

Table 1. Estimation Accuracy

\begin{tabular}{||c|c|c|c||}
\hline \multicolumn{2}{||c|}{} & SINGLE & BCA-LTE \\
\hline \multicolumn{2}{|c|}{ Accuracy } & $83.32 \%$ & $94.92 \%$ \\
\hline \multirow{2}{*}{ Inaccuracy } & Miss & $8.36 \%$ & $2.04 \%$ \\
\cline { 2 - 5 } & False alarm & $8.32 \%$ & $3.04 \%$ \\
\hline
\end{tabular}



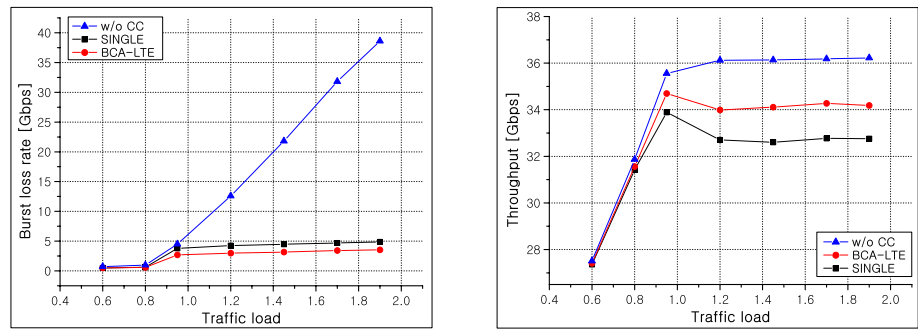

Fig. 3. (a) Burst loss rate (b) Throughput

The performance of three algorithms are compared in Fig. 3 in terms of burst loss rate and throughput. For the algorithm without burst congestion control, the burst loss rate keeps linearly increasing with the traffic load. It achieves a high throughput around $36 \mathrm{Gbps}$ at the cost of high burst loss rate. Two algorithms (SINGLE and BCA-LTE) well control the congestion. In particular, BCA-LTE keeps the burst loss rate lower than SINGLE (about 28\% gain), while achieving higher throughput. This also well supports the advantage of multiple statistics on estimation.

It is expected that BCA-LTE algorithm can enhance the stability of OBS network by proactively and accurately controlling the burst rate.

\section{Acknowledgement}

This work was supported by the Korea Science and Engineering Foundation (KOSEF) grant funded by the Korea government(MOST) (No. R11-2000-07401001-0).

\section{References}

1. C. Qiao and M. Yoo, "Optical Burst Switching (OBS) - A New Paradigm for an Optical Internet," Journal of High Speed Networks, Vol. 8, No. 1, pp. 69-84, Jan. 1999.

2. G. Thodime, V. M. Vokkarane and J. Jue, "Dynamic Congestion-Based Load Balanced Routing in Optical Burst-Switched Networks," In Proc. of Global Telecommunications Conference (GLOBECOM), Vol. 5, pp. 2628-2635, Dec. 2003.

3. Y. Gu, H. O. Wang, Y. Hong and L. G. Bushnell, "A Predictive Congestion Control Algorithm for High Speed Communication Networks," In Proc. of American Control Conference, 2001. 